\title{
Stat4 suppresses the proliferation of connective tissue-type mast cells
}

\author{
Tatsuki R Kataoka ${ }^{1,2}$ and Yasuko Nishizawa ${ }^{2,3}$
}

Mast cells are the progeny of hematopoietic stem cells, and murine mast cells are usually divided into two distinct populations, mucosal mast cells (MMCs) and connective tissue-type mast cells (CTMCs). We previously reported that CTMCs expressed signal transducer and activator of transcription (Stat) 4, but MMCs did not. Stat4 is also expressed in T cells and plays important roles in their homeostasis. In the present study, we show that Stat4 is involved in the homeostasis of CTMCs. The number of skin CTMCs increased in Stat4-deficient Balb/c mice, but that of gastric MMCs did not, when compared to those in control Balb/ $\mathrm{c}^{+/+}$mice. The comparison between cultured Stat4-deficient CTMCs and cultured Balb/c $\mathrm{c}^{+/+}$CTMCs revealed that cell cycle progression and cyclin D3 expression in the cultured Stat4-deficient CTMCs were enhanced in a Stat3 activation-dependent manner. This phenotype was explained by upregulation of KitL-induced interleukin (IL)-6 acting in an autocrine manner in cultured Stat4-deficient CTMCs. These results show that Stat4 suppresses the proliferation of CTMCs by controlling IL- 6 via an autocrine mechanism.

Laboratory Investigation (2008) 88, 856-864; doi:10.1038/labinvest.2008.51; published online 2 June 2008

KEYWORDS: IL-6; mast cell; Stat3; Stat4

\section{INTRODUCTION}

Mast cells are immunocompetent cells that are found in almost all tissues and function as sentinels of immune responses. ${ }^{1}$ They are descendents of hematopoietic stem cells ${ }^{2}$ and undergo terminal differentiation within the tissues in which they reside. ${ }^{3}$ Murine mast cells are usually classified as mucosal mast cells (MMCs) and connective tissue-type mast cells (CTMCs). ${ }^{1}$ This classification originally described the distribution of the cells. MMCs are located primarily in the gastrointestinal mucosa and lung bronchi, and CTMCs are widespread in all connective tissues, such as skin. However, many other differences exist between MMCs and CTMCs in addition to location. ${ }^{1,3}$ In terms of their cytoplasmic granules, MMCs have chondroitin sulfate-containing granules, whereas those of CTMCs are rich in heparin. ${ }^{1,3}$ In a previous study, we reported the differential expression of a transcription factor between these two types of mast cells, specifically that CTMCs expressed signal transducer and activator of transcription (Stat) 4, whereas MMCs did not. ${ }^{4}$

Interleukin (IL)-3 and Kit ligand (KitL) (also known as stem cell factor, steel factor, mast cell growth factor) play central roles in mast cell proliferation and survival. ${ }^{5-7}$ IL-3-dependent cultured mast cells (cultured MMCs) show an immature phenotype and are almost identical to MMCs. ${ }^{5,6}$ KitL-dependent cultured mast cells (cultured CTMCs) exhibit a CTMC-like phenotype. ${ }^{7}$ Co-culture of cultured MMCs with fibroblasts results in changes to a CTMC-like phenotype, because fibroblast-expressed KitL stimulates the cultured MMCs. ${ }^{8}$ IL-3 and KitL stimulation activates certain common signaling pathways, including Janus kinase (Jak)/Stat., ${ }^{9,10}$

Stat proteins are expressed in mast cells, and Jak/Stat signaling pathways are important for mast cell biology as well as for that of other immune cells. ${ }^{4-9-14}$ Stat3 activation is observed in human mastocytoma cells in which Kit bears a dominant active mutation. ${ }^{9}$ Stat $5 \mathrm{a} / \mathrm{Stat} 5 \mathrm{~b}$ is activated by both IL-3 and KitL stimulation, and mast cells cannot develop in Stat5a/Stat5b double-deficient mice. ${ }^{10}$ Stat 6 is involved in signaling with IL- $4,{ }^{11}$ another cytokine important for mast cell growth. Stat6-dependent IL-4 signaling in cultured MMCs suppresses expression of IL- $4,{ }^{11}$ FceRI, ${ }^{12} \mathrm{Kit}^{13}$ and TNF- $\alpha .{ }^{14}$ Stat 4 mediates IL-12 signaling and its deficiency

\footnotetext{
${ }^{1}$ Department of Pathology, Medical School/Graduate School of Frontier Bioscience, Osaka University, Osaka, Japan; ${ }^{2}$ Department of Pathology, Research Institute, Osaka Medical Center for Cancer and Cardiovascular Diseases, Osaka, Japan and ${ }^{3}$ Laboratory of Clinical and Experimental Pathophysiology, Graduate School of Pharmaceutical Science, Osaka University, Osaka, Japan

Correspondence: Dr TR Kataoka, MD, PhD, LAD/NIAID/NIH, Building 10, Room 11C206, 10 Center Drive, MSC 1881, Bethesda, MD 20892-1881, USA.

E-mail: kataokat@niaid.nih.gov
}

Received 25 February 2008; revised 29 April 2008; accepted 01 May 2008 
causes an impaired Th1 immune response. ${ }^{15}$ As stated above, Stat 4 is expressed in CTMCs and involved in their Th1 immune responses. ${ }^{4}$

In this report, we describe a novel role of Stat4 in CTMC. We found that Stat4-deficient Balb/c mice had a higher number of skin CTMCs than Balb/c $\mathrm{c}^{+1+}$ mice. We also demonstrated that cultured Stat4-deficient CTMCs showed enhanced cell cycle progression and increased cyclin D3 expression compared to cultured Balb/c $\mathrm{c}^{+/+}$CTMCs. These were due to increased Stat 3 activation in cultured Stat4deficient CTMCs by KitL-induced IL- 6 in an autocrine manner. This autocrine pathway has important roles in the increased number of tissue CTMCs in Stat4-deficient Balb/c mice. These data provide new insight into the role of Stat proteins in mast cells.

\section{MATERIALS AND METHODS Mice}

All animal care and experimentation were conducted in accordance with the Animal Care and Use Committee Guidelines of the Osaka Medical Center for Cancer and Cardiovascular Diseases or National Institute of Allergic and Infectious Diseases. Balb/c $\mathrm{c}^{+1+}$ mice were purchased from Japan SLC (Hamamatsu, Japan) or Jackson Laboratory (Bar Harbor, ME, USA), and Stat4-deficient Balb/c mice were purchased from Jackson Laboratory. ${ }^{15}$ Mice were maintained by repeated backcrosses to our own inbred colonies.

\section{Antibodies, Cytokines and Reagents}

Anti-cyclin D3 antibody (Ab) and anti-Stat3 Ab were obtained from Santa Cruz Biotechnology (Santa Cruz, CA, USA). Anti- $\beta$-tubulin $A b$ was purchased from Sigma Chemical (St Louis, MO, USA). Anti-phospho-Stat3 (Tyr705) Ab was purchased from Cell Signaling Technology (Beverly, MA, USA). Anti-IL-6 Ab (clone: MP5-20F3) and isotype control rat IgG I $_{1}$ clone: R3-34, 'control IgG') were acquired from BD Pharmingen (San Diego, CA, USA). The secondary Abs were peroxidase-labeled anti-rabbit or anti-mouse immunoglobulin (Ig) G antibodies (MBL, Nagoya, Japan). IL-3 (rmIL-3) was purchased from R\&D Systems (Minneapolis, MN, USA). Recombinant mouse KitL (rmKitL) was purchased from Peprotech (London, UK). STI571 (Imatinib) was purchased from Novartis Pharma AG (Basel, Switzerland). The specific Stat3 antisense oligodeoxynucleotide ('Stat3 decoy') and control oligodeoxynucleotide were described previously. ${ }^{16}$

\section{Culture of Mast Cells}

Mice (4-6 weeks of age) were used to obtain cultured mast cells. The mice were killed by decapitation following ether anesthesia and their spleens were removed. To prepare spleen cell suspensions, spleens were passed through a mesh. Spleen cells were cultured in $\alpha$-MEM supplemented with $10 \%$ fetal calf serum (FCS) and $10 \mathrm{ng} / \mathrm{ml} \mathrm{rmIL-3} \mathrm{for} 6$ weeks. ${ }^{4}$ Half of the medium was replaced every 5 days. More than $99 \%$ of non-adherent cells contained alcian blue-positive/safraninnegative granules, and were therefore considered to be cultured MMCs. In some experiments, spleen cells were cultured in $\alpha$-MEM supplemented with $10 \%$ FCS and rmKitL $(50 \mathrm{ng} / \mathrm{ml})$ for 8 weeks. ${ }^{4,7}$ Half of the medium was replaced every 5 days. Greater than $99 \%$ of non-adherent cells contained alcian blue-positive/safranin-positive granules and were therefore considered to be cultured CTMCs.

\section{Staining and Counting of Mast Cells}

At 6 weeks after birth, mice were killed by decapitation after ether anesthesia. Mast cell numbers in the skin, peritoneal cavity and glandular stomach were estimated, as described previously. ${ }^{17}$ In some experiments, we collected $1 \times 10^{4}$ non-adherent splenocytes derived from Balb/ $\mathrm{c}^{+/+}$or Stat4deficient mice after 2-week culture in KitL-contained medium $(50 \mathrm{ng} / \mathrm{ml})$, and re-suspend in KitL-contained medium $(50 \mathrm{ng} / \mathrm{ml})$ with or without imatinib $(1 \mu \mathrm{M}) .{ }^{18}$ Once a week, we counted the cell numbers for additional 6-week culture.

\section{Flow Cytometry}

Cultured CTMCs with or without exposure to anti-IL-6, control IgG, Stat3 decoy or mutant decoy, were washed, fixed and treated with RNase A. The cells were stained with $700 \mu \mathrm{M}$ propidium iodide for $30 \mathrm{~min}$ at room temperature. The cells were then analyzed using a FACScan flow cytometer (Becton Dickenson, Los Angeles, CA, USA), and the ratio of cells in S or G2/M phase using WinMDI was calculated (The Scripps Research Institute, La Jolla, CA, USA).

\section{Bromodeoxyuridine Cell Proliferation Assay}

Cultured MMCs with rmIL-3 (10 ng/ml) or cultured CTMCs with $\mathrm{rmKitL}(50 \mathrm{ng} / \mathrm{ml})$ were re-cultured for 1 day at $1.5 \times 10^{5}$ cells $/ 100 \mu \mathrm{l}$ in 96-well plates. Incorporation of bromodeoxyuridine (BrdU) into cultured MCs was assayed for using a BrdU cell proliferation assay kit (Calbiochem, San Diego, CA, USA), according to the manufacturer's instructions.

\section{Immunoblotting}

Whole-cell extracts of cultured MCs were obtained as described previously. ${ }^{4}$ The extracts were suspended in loading buffer, boiled, and analyzed by immunoblotting with anti-cyclin D3, anti- $\beta$-tubulin, anti-Stat3 or anti-phosphoStat3 (Tyr705) Abs and the corresponding secondary Abs. Protein levels were assayed with the ECL-plus detection kit (Amersham Life Sciences, Buckinghamshire, UK).

\section{ELISA}

Cultured MCs were re-cultured with rmKitL ( $50 \mathrm{ng} / \mathrm{ml}$ ) for 1 day at $1 \times 10^{5}$ cells $/ \mathrm{ml}$ in 24 -well plates. In some experiments, cultured CTMCs were exposed to an anti-IL-6 Ab overnight. The culture supernatants were assayed for IL-6 (R\&D Systems) using an ELISA kit, and phospho-Stat3 (Tyr705) in 
CTMCs was assessed using an ELISA kit (Cell Signaling), according to the manufacturer's instructions.

\section{Immunohistochemistry}

Skin tissues derived from Balb/c $\mathrm{c}^{+1+}$ or Stat4-deficient Balb/c mice were fixed in freshly prepared $4 \%$ paraformaldehyde in $0.1 \mathrm{~mol} / \mathrm{l}$ of phosphate buffer ( $\mathrm{pH} 7.4$ ) for $12 \mathrm{~h}$ at $4{ }^{\circ} \mathrm{C}$ Tissues were then dehydrated and embedded in paraffin. Serial sections ( $4 \mu \mathrm{m}$ thick) were cut. One of the section was stained with alcian blue and nuclear fast red to identify MCs, ${ }^{17}$ while the others were used for phospho-Stat3 staining. Immunoreacted cells to anti-phospho-Stat $3 \mathrm{Ab}$ were visualized with peroxidase and $0.05 \%$ diaminobenzidine- $0.02 \% \mathrm{H}_{2} \mathrm{O}_{2}$ solution, according to the manufacturer's instructions.

\section{Statistical Analysis}

Data were expressed as the means \pm s.e. Differences between groups were examined for statistical significance using Student's $t$-test (Excel; Microsoft, Redmond, WA, USA). A $P$-value less than 0.05 indicated statistical significance.

\section{RESULTS}

Increased Number of Tissue or Cultured CTMCs Derived from Stat4-Deficient Balb/c Mice and Enhanced Cell Cycle Transition in Cultured Stat4-Deficient CTMCs

First, we compared the number of tissue mast cells in Stat4deficient $\mathrm{Balb} / \mathrm{c}$ mice $v s$ that in control $\mathrm{Balb} / \mathrm{c}^{+/+}$mice histologically. The dorsal skin of Stat4-deficient Balb/c mice contained more CTMCs than that of control mice (Table 1 and Figure 1a). The percentage of peritoneal CTMC per peritoneal cell was significantly higher in Stat4-deficient $\mathrm{Balb} / \mathrm{c}$ mice than in control mice (Table 1), though the total numbers of peritoneal cells were comparable between Balb/ $\mathrm{c}^{+/+}$mice and Stat4-deficient Balb/c mice $\left(8.5 \pm 1.2 \times 10^{7}\right.$ cells per mouse vs $6.9 \pm 1.4 \times 10^{7}$ cells per mouse, $P>0.05$ ). The number of tissue MMCs in the mucosa of glandular stomach was comparable between Stat4-deficient Balb/c and control mice (Table 1 and Figure 1b). In contrast, the number of tissue CTMCs in the submucosa of glandular stomach was significantly higher in Stat4-deficient Balb/c mice than in control mice (Table 1).

Because KitL-dependent cultured mast cells are considered to have a phenotype similar to CTMCs, ${ }^{7}$ we next examined the proliferative phenotypes of KitL-dependent cultured mast cells derived from Balb/c $\mathrm{c}^{+1+}$ mice (cultured $\mathrm{Balb} / \mathrm{c}^{+/+}$ CTMCs) and those from Stat4-deficient Balb/c mice (cultured Stat4-deficient CTMCs). The morphological features or histamine content of cultured Balb/c $\mathrm{c}^{+1+}$ CTMCs and cultured Stat4-deficient CTMCs were essentially the same. The level of Kit mRNA or protein of cultured Stat4-deficient CTMC was comparable with that of cultured $\mathrm{Balb} / \mathrm{c}^{+/+}$ CTMC (data not shown). We also observed that the expression level of FceRI on cultured Balb/c ${ }^{+/+}$CTMC was comparable with that on cultured Stat4-deficient CTMC (data not shown).
Table 1 Number of mast cells in the stomach, the skin and the peritoneal cavity

\begin{tabular}{lccc}
\hline & $\begin{array}{c}\text { Balb/c } \\
\text { mice }\end{array}$ & $\begin{array}{c}\text { Stat4-deficient } \\
\text { Balb/c mice }\end{array}$ & $\begin{array}{c}\text { Number of } \\
\text { mice examined }\end{array}$ \\
\hline Mucosa of stomach $^{\mathrm{a}}$ & $122.9 \pm 14.6$ & $1133.8 \pm 6.4$ & 4 \\
Submucosa of stomach $^{\mathrm{b}}$ & $4.3 \pm 0.9$ & $11.8 \pm 1.5$ & 4 \\
Skin $^{c}$ & $157.9 \pm 10.2$ & $2241.7 \pm 20.8^{*}$ & 8 \\
Peritoneal cavity $^{\mathrm{d}}$ & $43.5 \pm 9.3$ & $559.4 \pm 13.1^{*}$ & 11 \\
\hline
\end{tabular}

\footnotetext{
${ }^{\mathrm{a}}$ Number of mast cells per $\mathrm{cm}$ of mucosa of glandular stomach.

${ }^{\mathrm{b}}$ Number of mast cells per $\mathrm{cm}$ of submucosa of glandular stomach.

${ }^{\mathrm{c}}$ Number of mast cells per $\mathrm{cm}$ of dorsal skin.

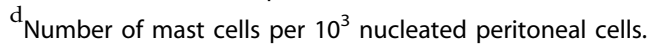

Mean \pm s.e. in the mice examined.

${ }^{*} P<0.05$ when compared to the value in Balb/ $\mathrm{c}^{+/+}$mice by $t$-test.
}

We counted the cell number of KitL-dependent splenocytes derived from Balb/c ${ }^{+/+}$or Stat4-deficient Balb/c mice. The cell numbers of both cultured Balb/c $\mathrm{c}^{+/+}$and Stat4-deficient splenocytes increased during the culture in KitL-contained medium. The increase in the number of cultured Stat4-deficient CTMCs was significantly greater than that of cultured Balb/c $\mathrm{c}^{+1+}$ CTMCs after 7 weeks from the start of the culture (Figure 2a). STI571 (Imatinib) is known as mouse Kit-KitL signal inhibitor. ${ }^{18}$ We used this inhibitor to elucidate the Kit-KitL signal effects on the CTMC numbers. The numbers of both $\mathrm{Balb} / \mathrm{c}^{+/+}$and Stat4-deficient splenocytes in the presence of STI571 gradually decreased. There was no significant difference between the number of Balb/ $\mathrm{c}^{+1+}$ cells and that of Stat4deficient cells in the presence of STI571 (Figure 2a). These results seemed to show that Kit-KitL-dependent proliferative signal was enhanced in Stat4-deficient CTMCs.

To examine proliferative phenotypes of CTMCs more closely, we examined the cell cycle status of these cells using flow cytometry and calculated the percentage of cells in the G2/M and S phases. The percentage of cultured Stat4-deficient CTMCs in the G2/M and S phases was significantly higher than that of cultured Balb/ $\mathrm{c}^{+/+}$CTMCs (Stat4deficient CTMCs $16.5 \pm 0.7 \%$ vs Balb/c ${ }^{+1+}$ CTMCs $9.3 \pm 0.9 \%$, $P<0.05$, Figure $2 \mathrm{~b})$. Stat 4 had an inhibitory effect on the KitL-dependent proliferation of CTMCs. In addition to KitL, IL-3 is important for mast cell proliferation. ${ }^{5-7}$ There was no difference between the percentage of cultured Stat4-deficient CTMCs in the G2/M and S phases and than that of cultured Balb/c $\mathrm{c}^{+1+}$ CTMCs with IL-3 stimulation (data not shown). We also examined incorporation of BrdU into cultured MMCs or CTMCs derived from Stat $4^{+/+}$or Stat $4^{-1-}$ Balb/c mice to assess the proliferative responses to KitL. BrdU will be incorporated into dividing cells. ${ }^{19}$ The incorporation of BrdU into cultured Stat4-deficient CTMCs with KitL 

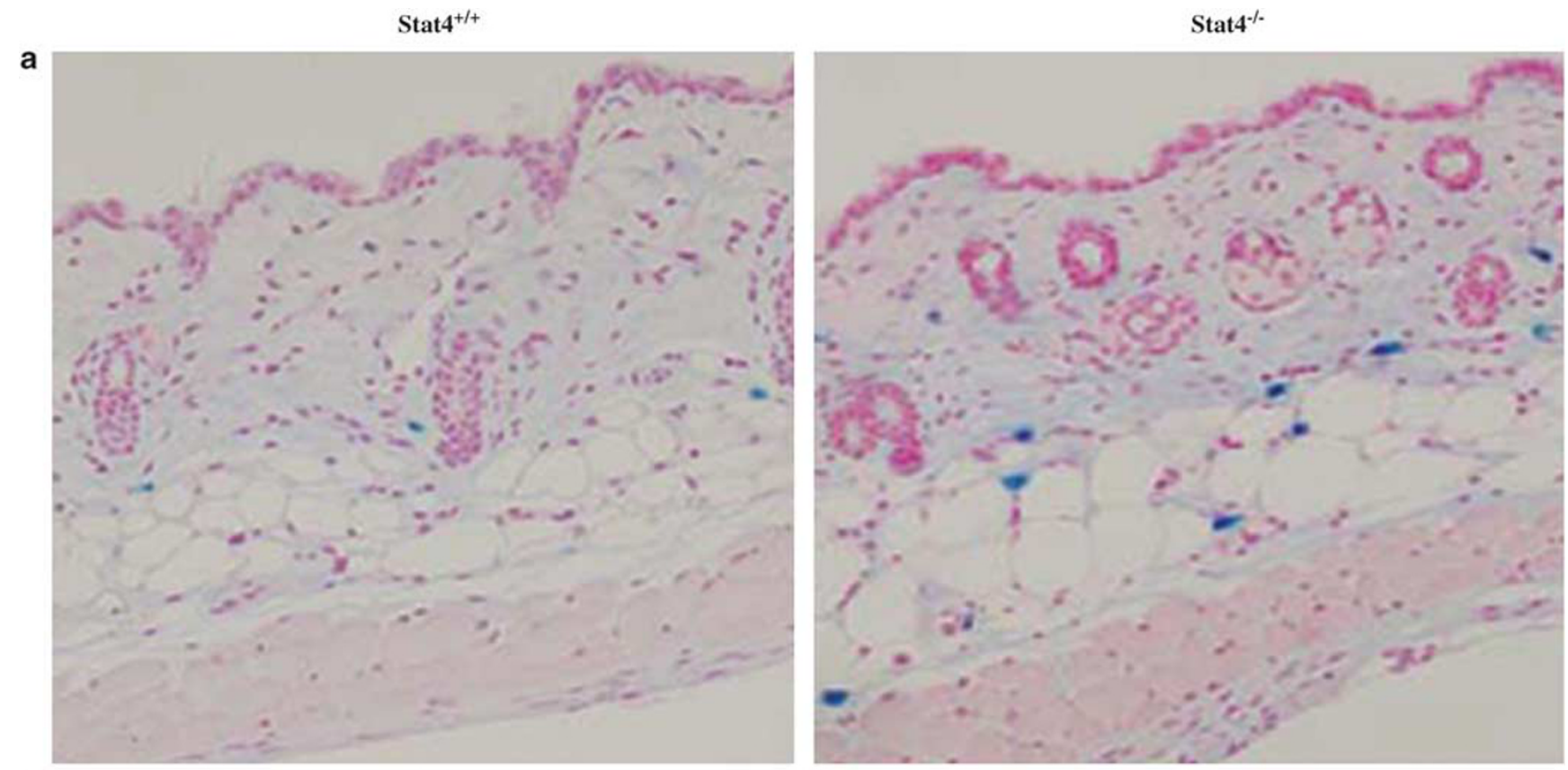

b
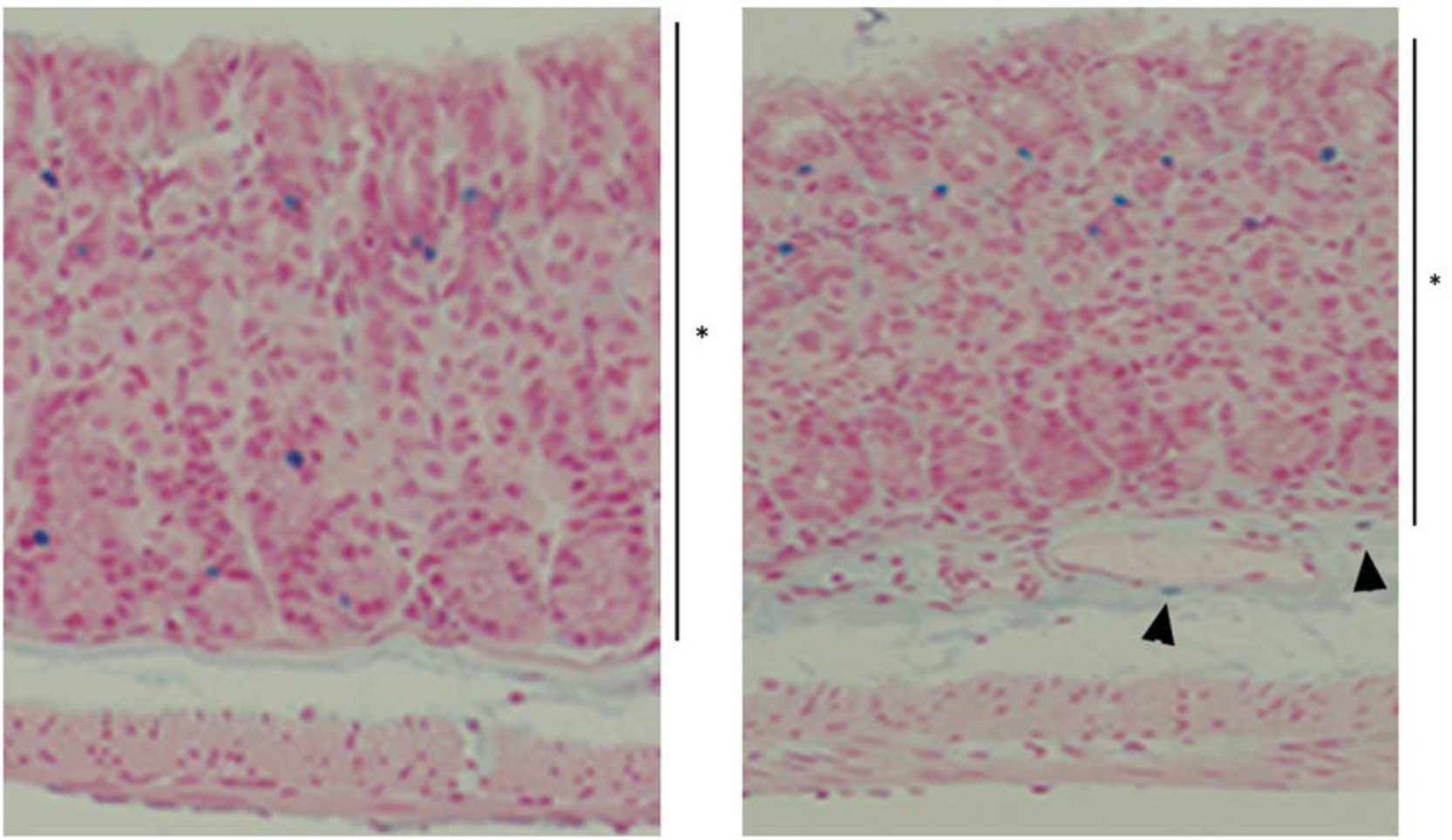

Figure 1 Skin and stomach histology of Balb/c ${ }^{+/+}$and Stat4-deficient Balb/c mice. (a) Skin and (b) stomach sections derived from 6-week-old Stat $4^{+1+}$ or Stat $4^{-1-}$ Balb/c mice. Mast cells are identified as blue-colored cells in the tissue. Tissue mast cells in the mucosa of glandular stomach $\left.{ }^{*}\right)$ were considered as MMCs. All of skin mast cells and submucosal mast cells in the glandular stomach (indicated by arrowheads) were considered as CTMCs. Data are representative of skin samples from eight individual mice or stomach samples from four individual mice.

stimulation was significantly higher than that into cultured Balb/c $\mathrm{c}^{+/+}$CTMCs, though there was no difference between the incorporation of cultured Stat4-deficient MMCs with IL-3 stimulation and that into cultured Balb/c $\mathrm{c}^{+/+}$MMCs (Figure 2c).

\section{Involvement of Stat3 in the Cell Cycle Progression and the Cyclin D3 Expression of Cultured Stat4-Deficient CTMCs}

Stat3 has been reported to be essential for the growth of cell lines with a gain-of-function-type Kit mutation. ${ }^{9,20-22}$ To 

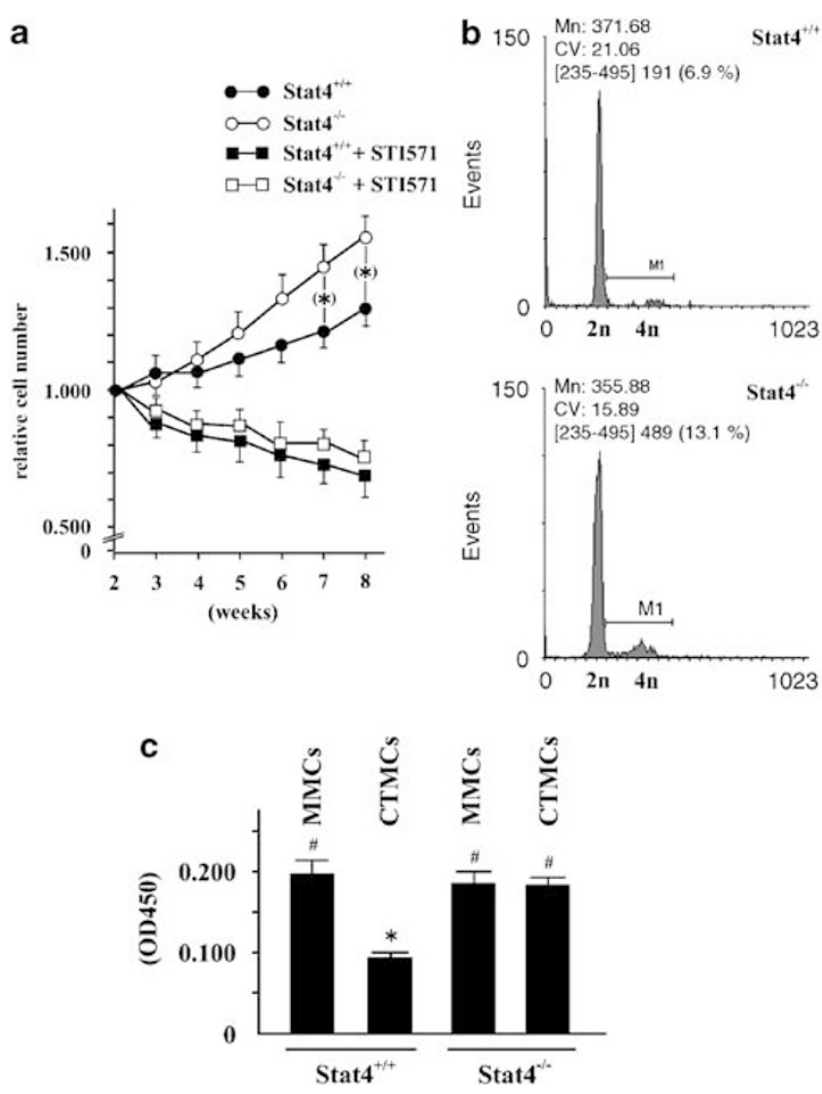

Figure 2 Increased number and enhanced cell cycle progression in cultured CTMCs derived from Stat4-deficient mice. (a) The cell numbers of cultured splenocytes derived from Stat $4^{+1+}$ or Stat $4^{-1-}$ Balb/c mice in KitL-contained medium with or without STI571 (Stat $4^{+/+}$mice; $n=5$, Stat $4^{-1-}$ mice; $\left.n=7\right) * P<0.05$ by $t$-test when compared to the value of Stat $4^{-/-}$Balb/c CTMCs to that of cultured Stat $4^{+/+}$CTMCs. (b) Cell cycle analyses of cultured CTMCs derived from Stat $4^{+/+}$or Stat $4^{-1-}$ Balb/c mice were conducted. Data are representative of 11 individual experiments. The gate referred to as ' $\mathrm{M} 1$ ' refers to $\mathrm{G} 2 / \mathrm{M}$ and $\mathrm{S}$ phases. (c) Incorporation of bromodeoxyuridine (BrdU) into cultured MMCs or CTMCs derived from Stat $4^{+/+}$or Stat $4^{-/-}$Balb/c mice. ${ }^{*} P<0.05$ by $t$-test when compared to the value of cultured Stat $4{ }^{+/+}$MMCs. ${ }^{~} P<0.05$ by $t$-test when compared to the value of cultured Stat $4^{+1+}$ CTMCs. examine whether the cell cycle progression in cultured Stat4deficient CTMCs was attributable to Stat 3 activation, we used the Stat3 decoy, a specific inhibitor of Stat3 signaling. ${ }^{16}$ Administration of the Stat 3 decoy dramatically decreased the percentages of cells in the G2/M and S phases of cultured $\mathrm{Balb} / \mathrm{c}^{+{ }^{+}}$and Stat4-deficient CTMCs (Table 2), demonstrating that Stat3 was indeed involved in the cell cycle progression of cultured CTMCs.

Cyclin D3 is rate limiting for G1- to S-phase transition and cell cycle progression in mast cells, ${ }^{23}$ and we found that Stat4 suppressed the expression of cyclin D3 in KitL-mediated cultured CTMC proliferation. We checked the protein level of cyclin D3 in cultured Balb/c $\mathrm{c}^{+/+}$or Stat4-deficient CTMCs by western blotting. The amount of cyclin D3 proteins in cultured Stat4-deficient CTMCs was larger than that of cultured Balb/c $\mathrm{c}^{+1+}$ CTMCs (Figure 3). Cyclin D3 expression level was correlated with the proportion of cultured CTMCs in $\mathrm{G} 2 / \mathrm{M}$ and $\mathrm{S}$ phases. Involvement of Stat3 in cyclin D3 expression has also been reported. ${ }^{24}$ This prompted us to analyze the Stat3 effect on protein levels of cyclin D3 in the cultured CTMCs. Western blotting analysis also revealed that cyclin D3 level was decreased in cultured Balb/c $\mathrm{c}^{+1+}$ and Stat4-deficient CTMCs, when Stat3 decoy was administrated (Figure 3).

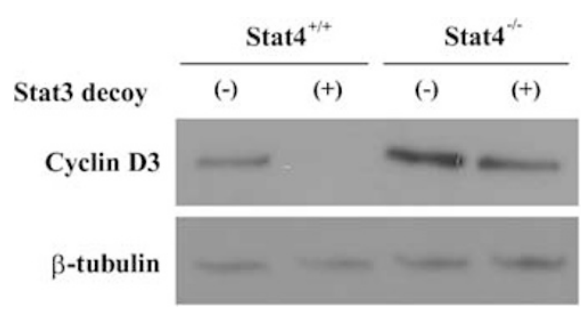

Figure 3 Cyclin D3 upregulation in cultured Stat4-deficient CTMCs by Stat3 activation. Western blot analyses were performed with cyclin D3 or $\beta$-tubulin antibodies in Stat $4^{+/+}$or Stat $4^{-/-}$Balb/c CTMCs cultured with Stat3 decoy ('Stat3 decoy $(+)^{\prime}$ ) or control decoy ('Stat3 decoy $\left.(-)^{\prime}\right)$. Data are representative from four individual experiments.

Table 2 Percentage of cultured CTMCs in the G2/M and S phases 1 day after administration of Stat3 decoy, neutralizing antibodies to IL-6 or Stat3 decoy with neutralizing antibodies to IL-6

$$
\text { Cultured Balb/c }{ }^{+/+} \text {CTMCs }
$$

$$
\begin{aligned}
& 10.3 \pm 0.5^{*} \\
& 5.4 \pm 1.3^{* \dagger} \\
& 9.0 \pm 0.9^{*} \\
& 4.9 \pm 1.6^{*, \dagger}
\end{aligned}
$$

Anti-IL-6

Stat3 decoy with anti-IL-6
Cultured Stat4-deficient Balb/c CTMCs

$$
\begin{aligned}
& 17.4 \pm 0.6^{\dagger} \\
& 12.7 \pm 0.8^{*, \dagger} \\
& 12.4 \pm 1.3^{*, \dagger} \\
& 12.2 \pm 1.7^{*, \dagger}
\end{aligned}
$$

Number of CTMCs examined

Mean (\%) \pm s.e. in the CTMCs examined.

${ }^{*} P<0.05$ when compared to the value of cultured Stat4-deficient Balb/c CTMCs without Stat3 decoy or neutralizing antibodies to IL-6 by $t$-test.

${ }^{\dagger} P<0.05$ when compared to the value of cultured Balb/ $\mathrm{c}^{+/+}$CTMCs without Stat3 decoy or neutralizing antibodies to IL- 6 by $t$-test. 
a

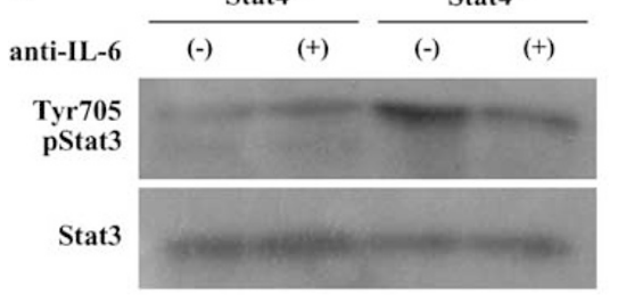

b

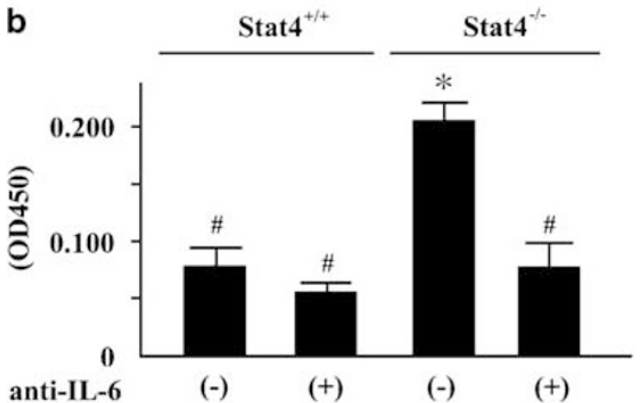

anti-IL-6

$(-)$

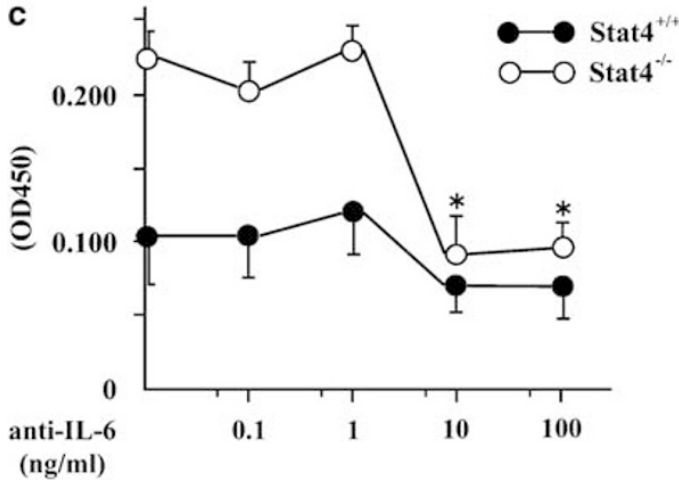

Figure 4 Stat3 activation of cultured Stat4-deficient CTMCs by IL-6 acting in an autocrine manner. (a) Western blot analyses were performed with Stat3 or Tyr705 phospho-Stat3 antibodies in Stat $4^{+/+}$or Stat $4^{-/-}$Balb/c CTMCs cultured with IL-6-neutralizing antibodies $(10 \mathrm{ng} / \mathrm{ml})\left(\right.$ 'anti-IL-6 $\left.(+)^{\prime}\right)$ or control lgG (10 ng/ml) ('anti-IL-6 $\left.(-)^{\prime}\right)$. Data are representative of three individual experiments. (b) Amounts of Tyr705 phospho-Stat3 in Stat $4^{+/+}$ or Stat $4^{-1-}$ CTMCs with IL-6-neutralizing antibodies $(10 \mathrm{ng} / \mathrm{ml})$ or control lgG $(10 \mathrm{ng} / \mathrm{ml})$ were analyzed by ELISA $(n=8)$. ${ }^{\star} P<0.05$ by $t$-test when compared to the value of cultured Stat ${ }^{+/+}$CTMCs with control IgG. ${ }^{\#} P<0.05$ by $t$-test when compared to the value of cultured Stat $4^{-1-}$ CTMCs with control IgG. (c) Amounts of Tyr705 phospho-Stat3 in Stat $4^{+/+}$ or Stat $4^{-1-}$ CTMCs with IL-6-neutralizing antibodies at the indicated concentrations were analyzed by ELISA $(n=4) .{ }^{*} P<0.05$ by $t$-test when compared to the value of cultured Stat $4^{+/+}$or Stat $4^{-1-}$ CTMCs without the IL-6-neutralizing antibodies.

\section{Enhanced Stat3 Activation in Cultured Stat4-Deficient CTMCs by Autocrine Actions of IL-6}

To examine the status of Stat 3 in cultured $\mathrm{Balb} / \mathrm{c}^{+/+}$and Stat4-deficient CTMCs, we examined the amounts of total Stat 3 and Tyr705 phospho-Stat3 by western blotting. The levels of total Stat3 were comparable between cultured Balb/c ${ }^{+1+}$ CTMCs and Stat4-deficient CTMCs (Figure 4a). In contrast, the amount of Tyr705 phospho-Stat 3 was higher in cultured Stat4-deficient CTMCs compared to cultured Balb/c ${ }^{+1+}$ CTMCs (Figure 4a).

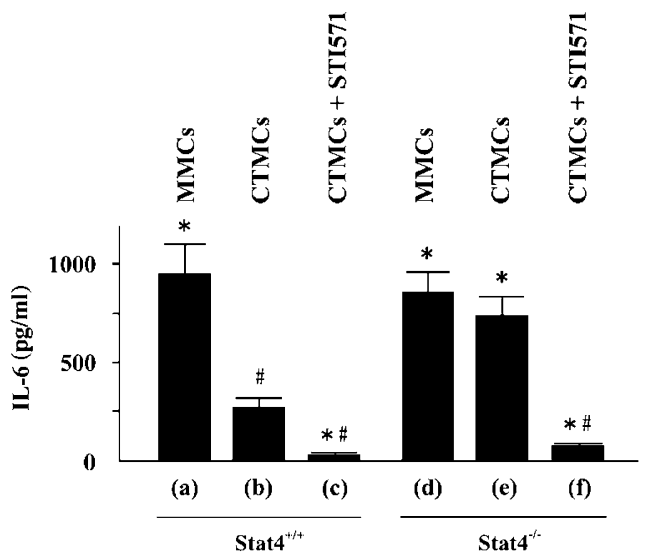

Figure 5 Enhanced IL-6 secretion from cultured Stat4-deficient CTMCs by KitL stimulation. IL- 6 secretion by cultured MMCs or CTMCs derived from Stat $4^{+/+}$or Stat $4^{-1-}$ Balb/c mice with or without STI571.

(a) Stat $4^{+/+}$MMCs without STI571, (b) Stat $4^{+/+}$CTMCs without STI571, (c) Stat $4^{+/+}$CTMCs with STI571, (d) Stat $4^{-/-}$MMCs without STI571, (e) Stat $4^{-1-}$ CTMCs without STI571, (f) Stat $4^{-1-}$ CTMCs with STI571. Culture supernatants of the indicated cultured mast cells with stimulation by KitL $(50 \mathrm{ng} / \mathrm{ml})$ for 1 day were analyzed by ELISA $(n=6) .{ }^{*} P<0.05$ by $t$-test when compared to the value of cultured Stat $4^{+/+}$CTMCs. ${ }^{\#} P<0.05$ by $t$-test when compared to the value of cultured Stat $4^{-1-}$ CTMCs.

KitL stimulation activates IL-6 secretion from cultured mast cells, ${ }^{25}$ and IL-6 is known to induce Stat3 phosphorylation at the Tyr705 residue. ${ }^{26}$ Next, we examined whether KitL-induced IL-6 had an effect on the phosphorylation of Stat 3 in cultured CTMCs and whether it occurred via an autocrine mechanism. Neutralizing antibodies against IL-6 $(10 \mathrm{ng} / \mathrm{ml})$ significantly lowered the amounts of Tyr705 phospho-Stat3 in cultured Stat4-deficient CTMCs (Figure 4a). In contrast, the amount of Tyr705 phospho-Stat3 in cultured $\mathrm{Balb} / \mathrm{c}^{+1+}$ CTMCs was not influenced by addition of the neutralizing antibodies (Figure 4a). ELISA analysis showed essentially similar results (Figure $4 \mathrm{~b}$ ). The inhibitory effect of neutralizing antibodies against IL-6 on the phosphorylation of Stat 3 was dose dependent (Figure 4c). These data showed that IL-6 induced Stat3 phosphorylation in cultured CTMCs by the autocrine manner.

The level of Tyr705 phospho-Stat3 was lower in cultured $\mathrm{Balb} / \mathrm{c}^{+1+}$ or Stat4-deficient MMCs in IL-3-contained medium when compared to cultured Balb/c $\mathrm{c}^{+/+}$CTMCs, and there was no difference between the amount of Tyr705 phospho-Stat 3 and that of cultured Balb/c ${ }^{+1+}$ CTMCs (data not shown).

We also examined the effect of IL- 6 neutralization on the cell cycle status of cultured Balb/c $\mathrm{c}^{+/+}$and Stat4-deficient CTMCs using flow cytometry. Anti-IL-6 antibodies significantly reduced the percentage of cultured Stat4-deficient CTMCs in the G2/M and S phases, but not that of cultured Balb/c $\mathrm{c}^{+1+}$ CTMCs (Table 2). In contrast, administration of anti-IL-6 antibodies with Stat 3 decoy declined the percentage of both cultured $\mathrm{Balb} / \mathrm{c}^{+/+}$and cultured Stat4-deficient CTMCs in the G2/M and S phases (Table 2), like the data of 

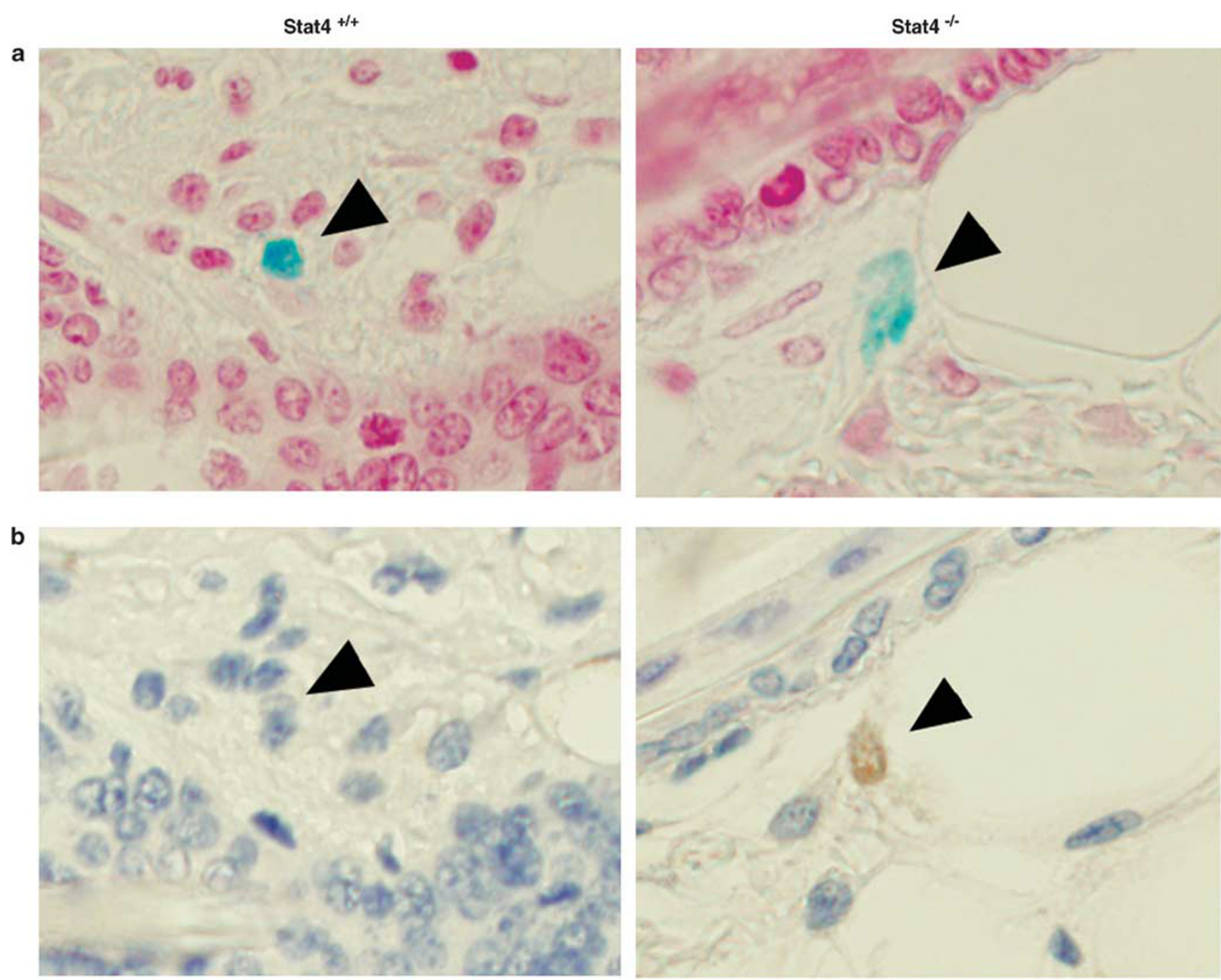

Figure 6 Phospho-Stat 3 immunostaining of skin mast cells derived from Stat $4^{+/+}$or Stat $4^{-/-}$Balb/c mice. (a) Alcian blue staining. Mast cells are identified as blue-colored cells in the tissue. (b) Immunostaining for phospho-Stat3 (Tyr705). Mast cells in the skin of Stat $4^{+/+}$or Stat4 ${ }^{-1-}$ Balb/c mice are indicated by arrowheads.

Stat3 decoy sole administration (Table 2). Taken together, administration of anti-IL-6 administration declined the percentage of cultured Stat4-deficient CTMCs in the G2/M and $S$ phases but did not that of cultured Balb/ $\mathrm{c}^{+/+}$CTMCs, though Stat3 decoy administration with or without anti-IL-6 antibodies did decrease the percentage of both cultured $\mathrm{Balb} / \mathrm{c}^{+/+}$and cultured Stat4-deficient CTMCs in the G2/M and $S$ phases. These data show that IL- 6 contributed to the cell cycle transition of cultured Stat4-deficient CTMCs in autocrine- and Stat3-dependent manners.

Next, we checked the amounts of IL- 6 produced by cultured Balb/ $\mathrm{c}^{+/+}$or Stat4-deficient CTMCs in KitL $(50 \mathrm{ng} / \mathrm{ml}$ )containing medium. Cultured Stat4-deficient CTMCs produced significantly larger amounts of IL- 6 than cultured $\mathrm{Balb} / \mathrm{c}^{+/+}$CTMCs (Figure 5). We incubated cultured $\mathrm{Balb} / \mathrm{c}^{+1+}$ or Stat4-deficient CTMCs with STI571, and found that STI571 suppressed IL-6 production from both types of CTMCs (Figure 5). These data suggest that Stat4 in CTMCs suppressed Kit-KitL signaling and downregulated IL-6 production. We also compared IL-6 production between cultured Balb/c $\mathrm{c}^{+1+}$ MMCs and cultured Stat4-deficient MMCs, but found no significant difference between them (Figure 5). This is compatible with the our previous finding that MMCs did not express a detectable level of Stat $4 .{ }^{4}$

\section{In Vivo Status of IL-6 and Stat3 of Stat4-Deficient Balb/c Mice}

To confirm our in vitro data, we checked IL-6 or Stat3 status in Stat4-deficient Balb/c mice. First, we measured the concentration of IL- 6 of the blood of Balb/c ${ }^{+/+}$or Stat4deficient mice. There was no significant difference between that of Balb/ $\mathrm{c}^{+1+}$ mice and that of Stat4-deficient mice 


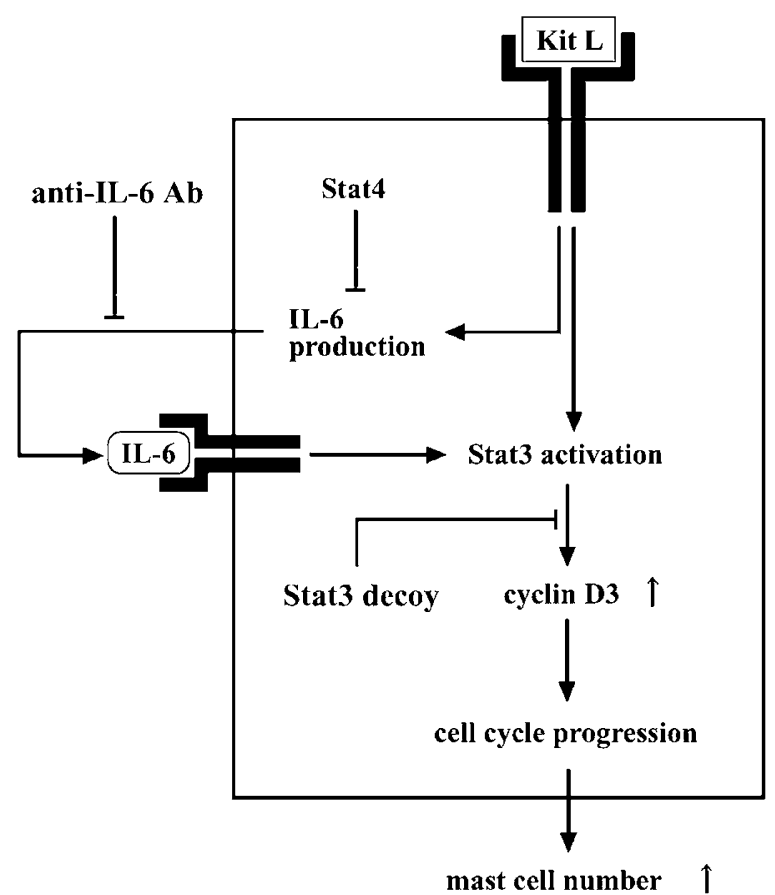

Figure 7 Current working model of CTMC proliferation. Stat 4 suppresses KitL-induced IL- 6 production. This model provides an explanation for the increased CTMC number in Stat $4^{-1-}$ Balb/c mice.

(data not shown). IL-6 derived from CTMCs seemed to function locally, but not systemically.

To evaluate the status of Stat3 in skin CTMCs of Balb/c $\mathrm{c}^{+/+}$or Stat4-deficient mice, we executed immunohistochemical stain by using anti-phospho-Stat3 (Tyr705) $\mathrm{Ab}$. We could not detect the positive staining for phosphoStat3 of skin CTMCs of Balb/ $\mathrm{c}^{+/+}$mice (Figure 6). In contrast, the phospho-Stat3 staining was seen in a part of skin CTMCs of Stat4-deficient Balb/c mice (Figure 6). The proportion of phospho-Stat3-positive CTMCs was $5.33 \pm 1.33 \%$ in the skin of Stat4-deficient Balb/c mice. These suggested that the frequency of Stat 3 activation in Stat4-deficient CTMCs was higher than that in Balb/ $\mathrm{c}^{+/+}$ CTMCs in vivo.

\section{DISCUSSION}

We found that Stat 4 influenced homeostasis of CTMCs, and observed that CTMC number increased in Stat4-deficient Balb/c mice (Figure 1, Table 1). Stat 4 effects on the proliferation of CTMCs explained this observation. We also tested the possibilities that Stat 4 had some influences on the numbers of mast cell progenitors, trafficking or survival of mast cells. In contrast to the increased number of tissue CTMCs, the numbers of mast cell progenitors of Stat4-deficient Balb/c mice decreased (data not shown), ${ }^{27}$ as is the case of other hematopoietic lineage. ${ }^{28}$ We could not find the difference in the trafficking or survival of cultured Balb/c $\mathrm{c}^{+/+}$ CTMCs and that of cultured Stat4-deficient CTMCs (data not shown; Figure 2a).
The present study showed that Stat 4 deficiency in CTMCs upregulated KitL-induced IL- 6 autocrine effects. And, there was a difference between the IL-6 neutralization influences on cultured Stat4-deficient CTMCs and those on cultured $\mathrm{Balb} / \mathrm{c}^{+/+}$CTMCs; IL-6 neutralization showed apparent effects on Stat 3 activation and cell cycle progression in cultured Stat4-deficient CTMCs but not those in cultured Balb/c $\mathrm{c}^{+1+}$ CTMCs (Table 2 and Figure $4 \mathrm{a}, \mathrm{b}$ and d). These results suggest that a certain level of IL- 6 is required for an optimal effect on Stat 3 activation or cell cycle progression of CTMCs. In contrast to IL-6 neutralization, Stat3 abrogation or Stat3 inhibition with IL-6 neutralization had a significant effect on the cell cycle progression of both cultured Balb/c $\mathrm{c}^{+/+}$and cultured Stat4-deficient CTMCs (Table 2). This difference would be explained by the contribution of Kit-mediated Stat3 phosphorylation in cultured CTMCs. KitL stimulation caused Tyr705 Stat3 phosphorylation in cultured MMCs, ${ }^{29}$ and the gain-of-function-type mutation in Kit also induced constitutive phosphorylation of Stat3 at the Tyr705 residue. ${ }^{22}$ It is conceivable that the Stat 3 decoy was thought to have inhibited both IL-6 receptor-mediated and Kit-mediated signal pathways.

This study showed the important roles of Stat3 in KitL-dependent mast cell proliferation as previously reported. ${ }^{9,20-22}$ Grb2-associated binder-2 (Gab2) is also important for KitL-dependent mast cell proliferation by activating the Rac/mitogen-activated protein kinase (MAPK) pathway. ${ }^{30,31}$ In addition, IL-6 activates both Stat3 and Gab/MAPK pathway. ${ }^{32}$ We did not detect the difference between the status of this signaling in cultured $\mathrm{Balb} / \mathrm{c}^{+/+}$ CTMCs and that in cultured Stat4-deficient CTMCs (data not shown). Stat 4 inhibited IL-6-Stat3 signaling, but did not inhibit IL-6-Gab/MAPK signaling.

We checked the effects of KitL administration on the status of Stat 4 in CTMCs. The phosphorylation of Stat 4 did not change in cultured CTMCs either with or without KitL. And, we also observed that Stat 4 distributed in the cytoplasm of CTMCs either with KitL or without KitL (data not shown). These suggested that Stat 4 did not locate in the downstream of Kit-KitL signals, though Stat4 modified KitL-dependent proliferative phenotypes of CTMCs.

Stat4 is important for IL-12 signaling, and Stat4-deficient $\mathrm{T}$ cells have diminished proliferative responses to IL-12. ${ }^{15,33}$ When we investigated whether the IL-12-Stat4 signal was involved in the proliferation of CTMC, IL-12 administration had no effect on the proliferation of cultured Balb/c $\mathrm{c}^{+/+}$or cultured Stat4-deficient CTMCs (data not shown). The proliferation of CTMC was not mediated by the IL-12-Stat4 signal, although Stat4 did mediate IL-12-induced IFN- $\gamma$ secretion from CTMCs. ${ }^{4}$

In summary, IL-6 induced Stat 3 activation in an autocrine manner; this plays a pivotal role in CTMC proliferation, and Stat 4 controls this pathway (summarized in Figure 7). Our present study thus provides new information on the role of the Stat protein in mast cell biology. 


\section{ACKNOWLEDGEMENT}

We thank Drs T Nakano (Osaka University) and S Koyasu (Keio University) for helpful discussion, and Mr M Kohara and Ms T Sawamura for technical assistance. This study was supported by grants from the Ministry of Education, Culture, Sports, Science and Technology, Japan.

\section{DISCLOSURE/DUALITY OF INTEREST}

The authors have no duality of interest.

1. Maurer $\mathrm{M}$, Theoharides $\mathrm{T}$, Granstein RD, et al. What is the physiological function of mast cells? Exp Dermatol 2003;12:886-910.

2. Kitamura $Y, G o ~ S$, Hatanaka K. Decrease of mast cells in $W / W^{v}$ mice and their increase by bone marrow transplantation. Blood 1978;52:447-452.

3. Nakano T, Sonoda T, Hayashi C, et al. Fate of bone marrow-derived cultured mast cells after intracutaneous, intraperitoneal, and intravenous transfer into genetically mast cell-deficient $\mathrm{W} / \mathrm{W}^{\mathrm{V}}$ mice. J Exp Med 1985;162:1025-1043.

4. Kataoka TR, Komazawa N, Morii E, et al. Involvement of connective tissue-type mast cells in Th1 immune responses via Stat4 expression. Blood 2005;105:1016-1020.

5. Nabel G, Galli SJ, Dvorak AM, et al. Inducer T lymphocytes synthesize a factor that stimulates proliferation of cloned mast cells. Nature 1981;291:332-334.

6. Galli SJ, Dvorak AM, Marcum JA, et al. Mast cell clones: a model for the analysis of cellular maturation. J Cell Biol 1982;95:435-444.

7. Tsai $M$, Takeishi $T$, Thompson $H$, et al. Induction of mast cell proliferation, maturation, and heparin synthesis by the rat c-kit ligand, stem cell factor. Proc Natl Acad Sci USA 1991;88: 6382-6386.

8. Levi-Schaffer F, Austin KF, Gravallese PM, et al. Coculture of interleukin 3-dependent mouse mast cells with fibroblasts results in a phenotypic change of the mast cells. Proc Natl Acad Sci USA 1986;83:6485-6488.

9. Fumo G, Akin C, Metcalfe DD, et al. 17-Allylamino-17-demethoxygeldanamycin (17-AAG) is effective in down-regulating mutated, constitutively activated KIT protein in human mast cells. Blood 2004;103:1078-1084.

10. Shelburne CP, McCoy ME, Piekorz R, et al. Stat5 expression is critical for mast cell development and survival. Blood 2003;102: 1290-1297.

11. Sherman MA, Secor VH, Lee SK, et al. STAT6-independent production of IL-4 by mast cells. Eur J Immunol 1999;29:1235-1239.

12. Ryan JJ, DeSimone S, Klisch G, et al. IL-4 inhibits mouse mast cell Fc\&RI expression through a STAT6-dependent mechanism. J Immunol 1998;161:6915-6923.

13. Mirmonsef $P$, Shelburne $C P$, Yeatman II $F$, et al. Inhibition of Kit expression by IL- 4 and IL-10 in murine mast cells: role of STAT6 and phosphatidylinositol 3'-kinase. J Immunol 1999;163:2530-2539.

14. Suzuki K, Nakajima $\mathrm{H}$, Ikeda $\mathrm{K}$, et al. IL-4-Stat6 signaling induces tristetraprolin expression and inhibits TNF- $\alpha$ production in mast cells. J Exp Med 2003;198:1717-1727.

15. Kaplan MH, Sun Y-L, Hoey T, et al. Impaired IL-12 responses and enhanced development of Th1 cells in Stat4-deficient mice. Nature 1996;382:174-177.
16. Leong $\mathrm{P}$, Andrews GA, Johnson DE, et al. Targeted inhibition of Stat3 with a decoy oligonucleotide abrogates head and neck cancer cell growth. Proc Natl Acad Sci USA 2003;100:4138-4143.

17. Morii $E$, Ito $A$, Jippo $T$, et al. Number of mast cells in the peritoneal cavity of mice. Am J Pathol 2004;165:491-499.

18. Chen H, Isozaki K, Kinoshita $\mathrm{K}$, et al. Imatinib inhibits various types of activating mutant KIT found in gastrointestinal stromal tumors. Int J Cancer 2003;105:130-135.

19. Porstmann T, Ternynck T, Avrameas S. Quantitation of 5-bromo-2deoxyuridine incorporation into DNA: an enzyme immunoassay for the assessment of the lymphoid cell proliferative response. J Immunol Methods 1985;82:169-179.

20. Ning Z-Q, Li J, Arceci RJ. Signal transducer and activator of transcription 3 activation is required for $\mathrm{Asp}^{816}$ mutant c-Kit-mediated cytokine-independent survival and proliferation in human leukemia cells. Blood 2001;97:3559-3567.

21. Corbin AS, Griswold IJ, La Rosee P, et al. Sensitivity of oncogenic KIT mutants to the kinase inhibitors MLN518 and PD180970. Blood 2004;104:3754-3757.

22. Casteran N, De Sepulveda P, Beslu N, et al. Signal transduction by several KIT juxtamembrane domain mutations. Oncogene 2003;22:4710-4722.

23. Itakura A, Miura $Y$, Hikasa $Y$, et al. Interleukin-3 and stem cell factor modulate cell cycle regulatory factors in mast cells: negative regulation of $\mathrm{p} 27^{\mathrm{Kip} 1}$ in proliferation of mast cells induced by interleukin-3 but not stem cell factor. Exp Hematol 2001;29: 803-811.

24. Fukada T, Ohtani T, Yoshida $Y$, et al. STAT3 orchestrates contradictory signals in cytokine-induced $\mathrm{G}_{1}$ to $\mathrm{S}$ cell-cycle transition. EMBO J 1998;17:6670-6677.

25. Gagari $\mathrm{E}$, Tsai $\mathrm{M}$, Lantz CS, et al. Differential release of mast cell interleukin-6 via c-kit. Blood 1997;89:2654-2663.

26. Kamiura D, Ishihara $\mathrm{K}$, Hirano T. IL-6 signal transduction and its physiological roles: the signal orchestration model. Rev Physiol Biochem Pharmacol 2003;149:1-38.

27. Morii E, Oboki K, Ishihara K, et al. Roles of MITF for development of mast cells in mice: effects on both precursors and tissue environments. Blood 2004;104:1656-1661.

28. Broxmeyer HE, Bruns HA, Zhang S, et al. Th1 cells regulate hematopoietic progenitor cell homeostasis by production of oncostatin M. Immunity 2002;16:815-825.

29. Shivakrupa R, Linnekin D. Lyn contributes to regulation of multiple Kitdependent signaling pathways in murine bone marrow mast cells. Cell Signal 2005;17:103-109.

30. Nishida K, Wang L, Morii E, et al. Requirement of Gab2 for mast cell development and KitL/c-kit signaling. Blood 2002;99: 1866-1869.

31. Yu M, Luo J, Yang W, et al. The scaffolding adapter Gab2, via Shp-2, regulates Kit-evoked mast cell proliferation by activating the Rac/JNK pathway. J Biol Chem 2006;281:28615-28626.

32. Nishihara $M$, Ogura $H$, Ueda $N$, et al. IL-6-gp130-STAT3 in T cells directs the development of IL-17+ $T_{h}$ with a minimum effect on that of Treg in the steady state. Int Immunol 2007;19:695-702.

33. Shen R, Kaplan MH. The homeostasis but not the differentiation of T cells is regulated by p27 $7^{\text {Kip } 1 . ~ J ~ I m m u n o l ~ 2002 ; 169: 714-721 . ~}$ 